> L'insulino-résistance, qui caractérise le diabète de type 2 et l'obésité, est due à une diminution de l'action de l'insuline sur ses tissus cibles (foie, tissu adipeux, muscles squelettiques). II est maintenant bien documenté qu'au niveau de ces tissus, l'accumulation ectopique d'acides gras, et en particulier de métabolites dérivés de ces acides gras, comme les céramides, joue un rôle crucial dans l'altération du message insulinique. Cette revue décrit le rôle majeur joué par les céramides dans le développement de l'insulino-résistance des tissus périphériques. <

Une épidémie mondiale d'obésité et de diabète se propage chez l'homme depuis plusieurs décennies. Selon l'Organisation mondiale de la santé (OMS), 422 millions de personnes étaient atteintes de diabète en 2014, contre 108 millions en 1980. Selon les estimations, en 2045, le nombre de diabétiques devrait atteindre au moins 629 millions [1] $(\rightarrow)$. Le diabète est une maladie $(\rightarrow)$ Voir le Faits et chiffres de C. Franc, $m / s n^{\circ} 8-9$, août-septembre 2013, page 711 caractérisée par une hyperglycémie chronique. Après plusieurs années de diabète non équilibré, des complications altérant la qualité de vie des patients apparaissent et peuvent entraîner une mort prématurée. Ces complications sont classées en deux groupes: des complications microvasculaires, telles que la rétinopathie, la neuropathie et la néphropathie; et des complications macro-vasculaires, telles que l'accident vasculaire cérébral, l'infarctus du myocarde ou l'artérite des membres inférieurs [2].

II existe deux principaux types de diabète. Le diabète de type 1 (DT1), qui représente $10 \%$ des cas de diabète, résulte d'un déficit total en insuline (insulinopénie) consécutif à la destruction auto-immune des cellules productrices d'insuline, les cellules $\beta$ pancréatiques. Le diabète de type 2 (DT2), le diabète le plus courant

Vignette (Photo (C)Inserm-Annicotte, Jean-Sébastien).

\section{Céramides, acteurs cruciaux dans le développement de l'insulino- résistance et du diabète de type 2}

Sophie Tan-Chen ${ }^{1,2}$, Olivier Bourron ${ }^{1-3}$, Éric Hajduch ${ }^{1,2}$

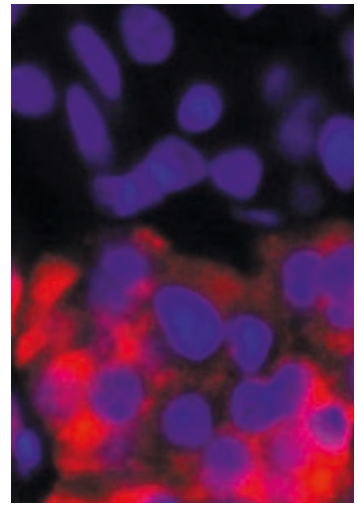

(90\% des cas de diabète), se carac-
${ }^{1}$ Centre de Recherche des Cordeliers, Inserm, Sorbonne Université, Université de Paris, 18 rue de l'École de Médecine, F-75006 Paris, France.

${ }^{2}$ Institut Hospitalo-Universitaire ICAN, Paris, France.

${ }^{3}$ Sorbonne Université, Assistance Publique-Hôpitaux de Paris, Service de Diabétologie et Maladies Métaboliques, Hôpital Pitié-Salpêtrière, 75013 Paris, France.

eric.hajduch@crc.jussieu.fr térise par deux dysfonctionnements cellulaires majeurs: une résistance à l'insuline d'organes périphériques, tels que le foie, le tissu adipeux et les muscles squelettiques, et un défaut de sécrétion de l'insuline plus ou moins marqué. La résistance à l'insuline entraîne une incapacité des tissus sensibles à l'insuline à répondre au signal de I'hormone. Le DT2 est associé à de nombreux gènes de susceptibilité dont l'expression dépend de facteurs environnementaux, tels que le niveau d'activité physique ou les régimes hyper-glucidiques et riches en graisse [3]. Dès que la résistance à l'insuline apparaît, le pancréas augmente physiologiquement sa production de l'hormone pour maintenir une glycémie normale. Une hyperglycémie s'observe lorsque la fonction de sécrétion d'insuline du pancréas devient insuffisante pour contrebalancer l'augmentation de la résistance à l'insuline des tissus périphériques. Ce dysfonctionnement de sécrétion d'insuline est lié à des facteurs génétiques / épigénétiques et environnementaux [4]. Plusieurs mécanismes ont été proposés pour expliquer à la fois la résistance à l'insuline tissulaire et les défauts de sécrétion d'insuline observés dans le DT2. Parmi ceux-ci, la lipotoxicité, la glucotoxicité, l'inflammation systémique de bas grade, le stress oxydatif et le stress du réticulum endoplasmique $[5,6]$ correspondent à différents mécanismes cellulaires qui peuvent converger vers une voie commune induisant une résistance à l'insuline.

Dans cette revue, nous nous concentrerons sur le rôle de la toxicité lipidique cellulaire, c'est-à-dire la lipotoxicité, et sur le développement de l'insulino-résistance tissulaire. 


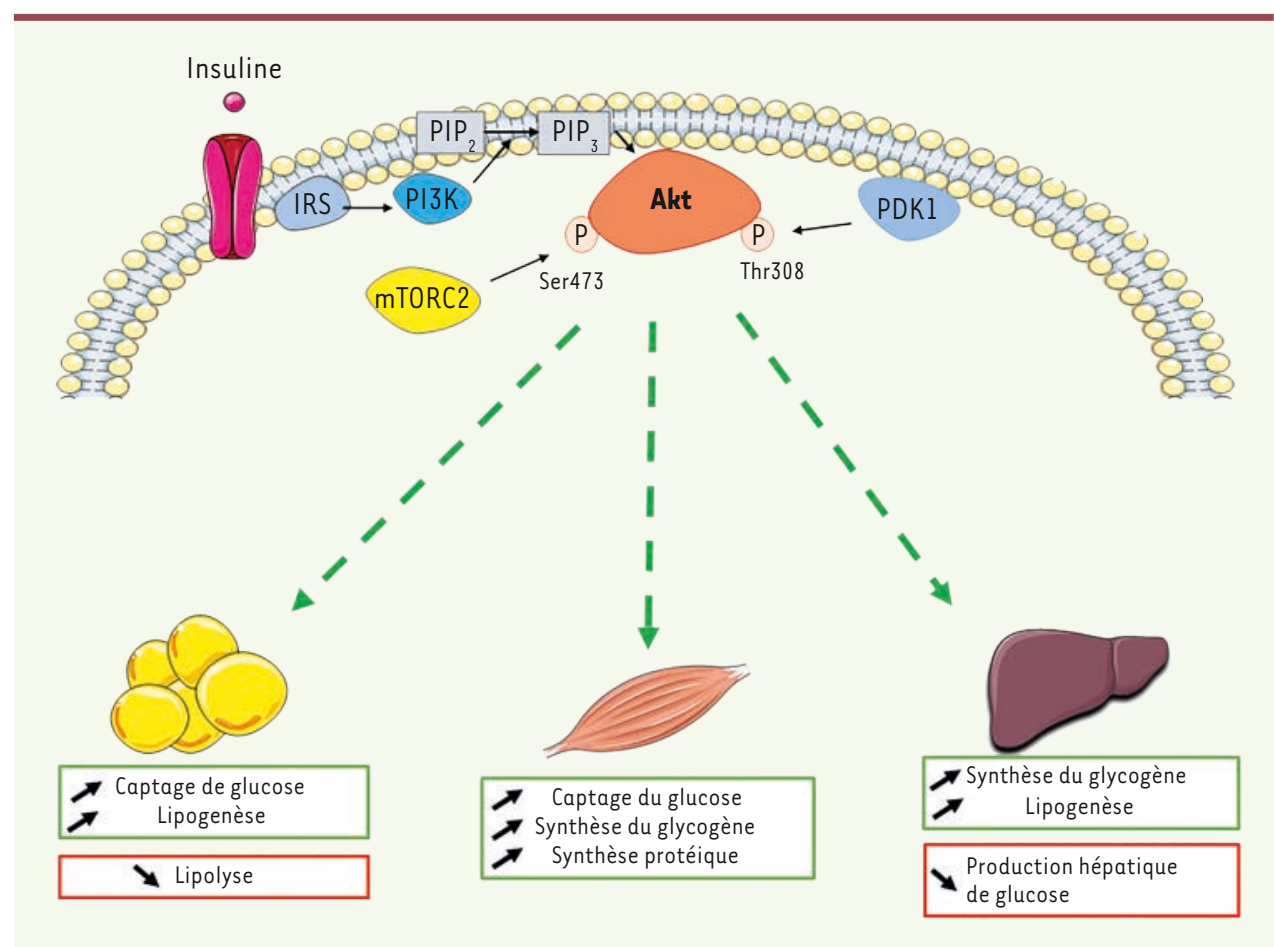

Figure 1. Voie de signalisation et rôle métabolique de l'insuline. L'insuline se lie à son récepteur qui active les IRS (insulin receptor substrates), la PI3K (phosphoinositol-3-kinase) et Akt (protéine kinase $B$ ) qui relaie les effets métaboliques de l'insuline et régule l'homéostasie glucidique dans les tissus insulino-sensibles. PIP2 : phosphatidylinositol 4,5-biphosphate. PIP3 : phosphatidylinositol 3,4,5 triphosphate; PDK1 : 3-phosphoinositide-dependent protein kinase-1 ; mTORC2 : mammalian target of rapamycin complex 2.

\section{La lipotoxicité}

Comme le muscle squelettique représente $40 \%$ de la masse corporelle humaine, et qu'il est quantitativement le tissu le plus important pour la capture du glucose en réponse à l'insuline, il est considéré comme la principale cible cellulaire dans le développement de la résistance à l'insuline. La plupart des études portant sur les mécanismes de résistance à l'insuline induite par lipotoxicité ont donc été réalisées principalement dans les tissus musculaires.

En 1963, Randle et ses collaborateurs ont postulé qu'une compétition entre le glucose et les acides gras, pour leur oxydation et leur absorption, était responsable de l'apparition d'une résistance à l'insuline dans le muscle et le tissu adipeux [7]. Des études réalisées in vivo chez des rongeurs et chez l'homme ont confirmé la relation entre lipides et résistance à l'insuline, mais elles ont également montré que, contrairement à l'hypothèse initiale de Randle, la résistance à l'insuline induite par les lipides n'était pas secondaire à une inhibition de la glycolyse [5]. En effet, les lipides agissent directement sur la signalisation insulinique en bloquant la translocation vers la membrane plasmique, en réponse à l'hormone, du transporteur de glucose sensible à l'insuline, GLUT4, réduisant ainsi l'entrée de glucose dans les cellules [5]. Chez l'homme, des données montrent clairement une forte corrélation entre le contenu lipidique intramusculaire et une résistance des cellules à l'insuline [8].

Le foie est un autre organe important impliqué dans la résistance à l'insuline. Comme dans le muscle, il semble également qu'une accumulation lipidique hépatique, que l'on appelle stéatose hépatique non alcoolique (NAFLD pour nonalcoholic fatty liver disease), soit associée à une résistance à l'insuline hépatique [9].

\section{Signalisation insulinique et fonctions métaboliques}

La première étape de l'activation de la voie de signalisation de l'insuline est sa liaison à son récepteur membranaire qui provoque l'autophosphorylation des sous-unités $\beta$ du récepteur [10]. Ainsi phosphorylé, le récepteur de l'insuline sera à l'origine de l'activation des IRS (pour insulin receptor substrate) qui, ensuite, activeront la PI3K (phosphatidylinositol-3-kinase) (Figure 1). Cette kinase génèrera alors des phospholipides membranaires spécifiques, indispensables au recrutement d'une autre kinase, Akt (protéine kinase B) $[10,11]$

$\rightarrow$ Voir la Nouvelle de E. Hajduch, $m / s n^{\circ} 10$, $(\rightarrow)$. octobre 2001, page 1084

En présence d'insuline, Akt est ainsi recrutée à la membrane plasmique des cellules où elle sera activée par phosphorylation sur deux sites critiques: la thréonine 308 (T308), dans sa boucle d'activation, et la sérine 473 (\$473), dans son motif hydrophobe (Figure 1). C'est la PDK1 (phosphoinositide-dependent kinase 1) qui phosphoryle Akt sur son site T308, et le complexe mTORC2 (mammalian target of rapamycin-2) qui phosphoryle son site S473 (Figure 1) [10].

Akt agit donc comme un relai de nombreuses activités métaboliques de l'insuline dans les différents tissus qu'elle cible. En effet, l'insuline stimule l'absorption du glucose dans les muscles squelettiques et les adipocytes, elle induit la synthèse de glycogène et son 
stockage dans les muscles et le foie, et la synthèse protéique dans les muscles; elle inhibe également la lipolyse adipocytaire et la production hépatique de glucose (Figure 1) [12].

\section{Les céramides et l'insulino-résistance}

Dans les situations d'obésité et le diabète de type 2, tous deux caractérisés par une résistance à l'insuline, l'accumulation ectopique d'acides gras est accrue en raison d'une augmentation de leur entrée dans les cellules et d'une réduction de leur oxydation [13]. Une corrélation inverse existe entre l'augmentation intracellulaire des concentrations d'acides gras et la sensibilité à l'insuline musculaire, hépatique et adipocytaire [14, 15]. Les céramides constituent les principaux lipides qui jouent un rôle crucial dans la résistance à l'insuline. Dans des cellules de mammifères, les céramides sont des lipides centraux du métabolisme des sphingolipides. Ils peuvent être à la fois lipides de signalisation, mais aussi précurseurs d'autres sphingolipides bioactifs, ceux-ci allant des glycosphingolipides complexes à des lipides «simples » comme les céramides-1-phosphate, les sphingomyélines (SM), les sphingosines et les sphingosines-1-phosphate [12].

\section{Voies de biosynthèse des céramides}

Les céramides sont largement distribués dans les membranes cellulaires où ils jouent un rôle structurel important. Ils participent également à la signalisation intracellulaire et à la régulation de la croissance, de la migration, de l'apoptose et de la différenciation cellulaires [16]. Les céramides sont constitués d'une base sphingoïde à longue chaîne qui est liée à un acide gras par une liaison amide. Ils peuvent être produits par l'hydrolyse de la sphingomyéline par des sphingomyélinases [17], ou par la dégradation de sphingolipides complexes localisés dans les lysosomes [17].

Dans un contexte d'obésité associée à une surcharge en acides gras, les céramides sont principalement produits de novo, à partir d'acides gras saturés (en particulier le palmitate) (Figure 2). Cette synthèse, qui s'effectue dans le réticulum endoplasmique ( $R \varepsilon)$, est le résultat de différentes réactions qui commencent par la condensation de la $L$-sérine avec le palmitoyl-CoA. Cette réaction est catalysée par la sérine palmitoyl-transférase (SPT) et génère le 3-keto-dihydrosphingosine, qui sera réduit en dihydrosphingosine par la 3-keto-dihydrosphingosine réductase. La dihydrosphingosine est ensuite $\mathrm{N}$-acylée par différentes isoformes de céramide synthases (CerS) pour former des dihydrocéramides. Chez les mammifères, six isoformes de CerS sont exprimées, CerS 1 à 6 [18]. Ces enzymes catalysent la même réaction chimique, mais elles présentent des caractéristiques spécifiques quant à la longueur des chaînes d'acyl-CoA qu'elles utilisent pour la N-acylation. C'est donc de la nature des isoformes de CerS que dépend la composition en acides gras des céramides. La chaîne carbonée de la partie sphingoïde, constituant la dihydrocéramide, sera finalement désaturée par la dihydrocéramide désaturase (DES1) pour former un céramide [17] (Figure 2).

\section{Céramide et lipotoxicité}

Une des premières études ayant analysé les concentrations intracellulaires en céramides chez des rats obèses insulino-résistants (rat Zucker $\mathrm{fa} / \mathrm{fa}$ ) a été celle réalisée par de Turinsky et al. Ces auteurs ont en effet constaté que ces rats présentaient une augmentation des concentrations intramusculaires et hépatiques en céramide $[19,20]$. Une telle augmentation a également été détectée dans d'autres modèles d'insulino-résistance (des souris ob/ob, souris nourries sous régime gras et rats perfusés avec des émulsions de lipides (les intra-lipides) [21]. Dans leur ensemble, ces études révèlent ainsi l'existence d'une relation inverse entre concentrations musculaires et hépatiques de céramides et sensibilité à l'insuline dans ces tissus. Des études réalisées, chez l'homme, ont confirmé cette relation inverse entre accumulation de céramides intramusculaires et sensibilité à l'insuline (pour revue voir [22]) [23-25] et le groupe de Goodpaster a montré que l'exercice physique permettait de réduire les concentrations musculaires de céramides chez les sujets obèses et résistants à l'insuline, avec une amélioration de leur sensibilité à l'insuline [26].

De nombreuses études ont mis en évidence le rôle crucial des céramides musculaires dans l'apparition de l'insulino-résistance et du DT2 [12]. Comme dans les muscles, l'accumulation de céramides dans les adipocytes est également corrélée à une résistance à l'insuline tissulaire [27]. Cette relation a été confirmée in vitro en utilisant des myotubes différenciés de souris (lignée C2C12), de rats (lignée L6) et de sujets humains, et des adipocytes. En effet, l'exposition de ces cellules à un acide gras saturé (le palmitate) ou à des céramides inhibe l'activation par l'insuline de la synthèse du glycogène et du transport du glucose [12].

Ces études révèlent donc une association entre la résistance à l'insuline et une augmentation des concentrations en céramides dans les muscles squelettiques et les adipocytes. Néanmoins, malgré l'abondante littérature mettant en évidence un lien entre céramides et insulino-résitance musculaire, quelques études rapportent des résultats divergents, avec l'absence de modification des concentrations des céramides musculaires en réponse à une perfusion lipidique, aussi bien chez des rats [28] que chez des sujets humains sains [29], ou obèses [30].

Le rôle des céramides dans l'apparition de la résistance à l'insuline hépatique est plus récent et il reste encore discuté. En effet, certaines études ne constatent aucune accumulation de céramides dans le foie gras $[31,32]$, rendant improbables ces lipides comme médiateurs de la résistance à l'insuline hépatique. 


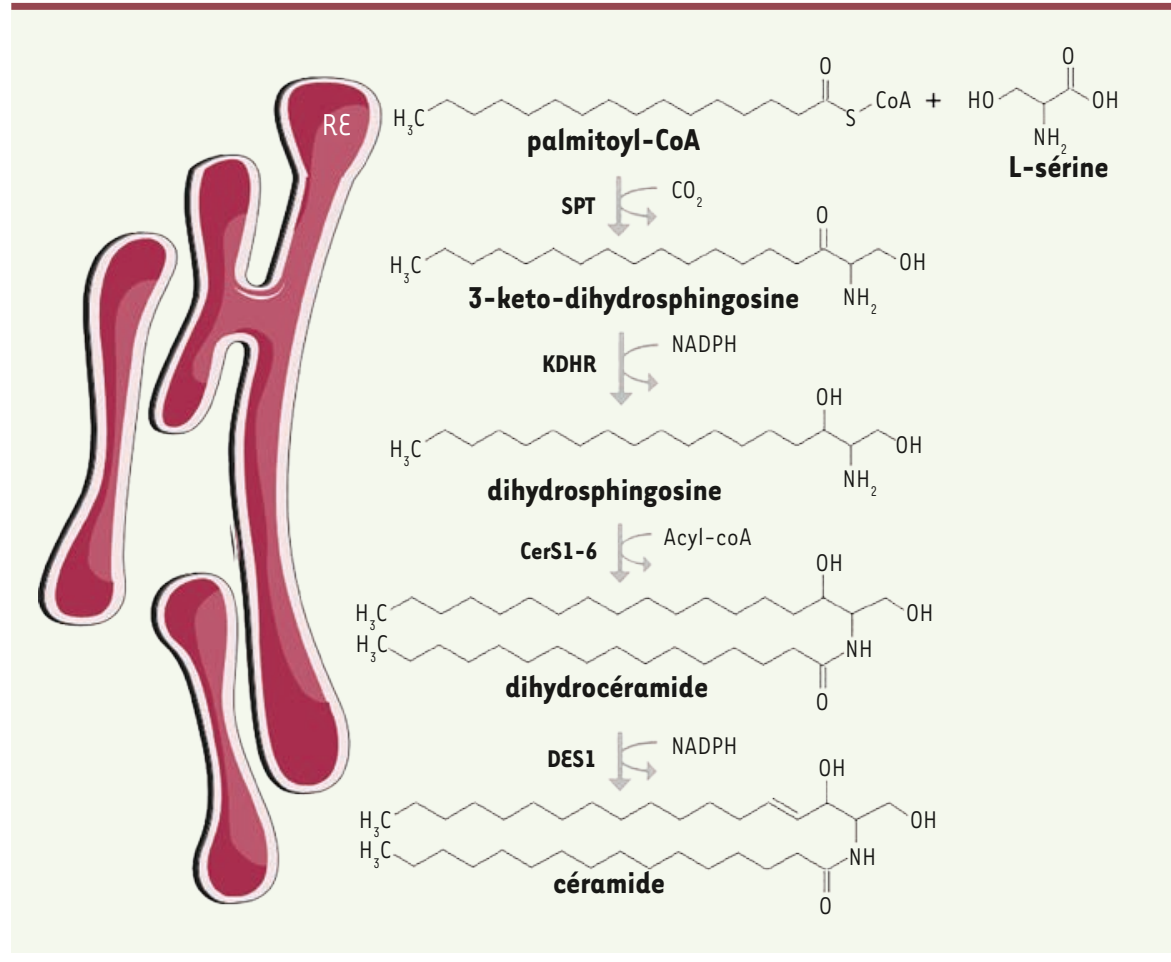

Figure 2. Voie de synthèse de novo des céramides. Le palmitate est l'acide gras saturé préférentiellement utilisé pour la synthèse de novo des céramides qui est réalisée dans le réticulum endoplasmique (RE) des cellules. Le palmitoyl-CoA est d'abord condensé avec une sérine pour former une 3-keto-dihydrosphingosine sous l'action de la sérine palmitoyl transférase (SPT). La 3-ketodihydrosphingosine est ensuite métabolisée en dihydrosphingosine par la 3-keto-dihydrosphingosine réductase (KDHR). La dihydrosphingosine formée est acylée par différentes isoformes de céramides synthases (CerS1-6) pour former des dihydrocéramides de différentes longueurs de chaîne. Les dihydrocéramides sont ensuite désaturés par la dihydrocéramide $\Delta 4$-désaturase (DESl) pour donner des céramides.

Néanmoins, plusieurs études récentes montrent une implication des céramides hépatiques dans l'apparition de la stéatose et de la résistance hépatique à l'insuline [33-38].

Selon le tissu étudié, des espèces différentes de céramides peuvent être impliquées. L'expression des différents isoformes de CerS, responsables de la $\mathrm{N}$-acylation de la base sphingoïde, varie en effet en fonction des tissus [18]. Par exemple, en conditions physiologiques, dans les muscles squelettiques, l'expression de CerSl est majoritaire, entraînant la biosynthèse prépondérante de céramides à longue chaîne C18-céramides ${ }^{1}$ [18]. Dans le foie, c'est l'expression de CerS2 qui prévaut, et des céramides à très longue chaîne, C22- et C24-céramides, sont majoritairement produits [18]. En condition lipotoxique, l'expression tissulaire des CerS peut évoluer, induisant un changement des espèces de céramides produites. Ainsi, une augmentation de l'expression de CerS6 a été observée dans le foie de souris obèses et insulinorésistantes, à l'origine d'une modification des concentrations hépatocytaires des différentes espèces céramides, avec une augmentation significative des concentrations de C16-céramides [39] dont plusieurs études ont montré la nocivité vis-à-vis de la sensibilité à l'insuline $[33,35,39]$. Les espèces de céramides qui induisent une résistance à l'insuline dans les cellules musculaires ne sont cependant pas encore clairement identifiées, même si l'accumulation de C18-céramides est souvent liée à une insulino-résistance $[40,41]$. Au contraire des céramides à chaîne courte, les espèces de céramides à chaîne longue plus ou moins insaturée (C22, C24:1 et C24) semble avoir une action protectrice sur la réponse à l'insuline [33].

${ }^{1} \mathrm{C} 18$ correspond au nombre de carbone formant la chaîne lipidique.

\section{Mécanisme d'action des céramides}

Les mécanismes par lesquels les céramides agissent sur la voie de signalisation insulinique dans les tissus sensibles à l'hormone sont maintenant établis, en particulier dans les cellules musculaires et adipocytaires (ils le sont moins au niveau hépatique). Dans ces cellules, ils inhibent spécifiquement deux acteurs cette voie de signalisation: Akt et les IRS. Les céramides activent rapidement la protéine kinase $C$ atypique $\mathrm{PKC \zeta}$ qui interagit avec le domaine $\mathrm{PH}$ d'Akt, homologue de la pleckstrine, et le phosphoryle sur un résidu Thr34/Ser34. Cette phosphorylation d'Akt est à l'origine de sa sa séquestration dans des domaines spécialisés de la membrane plasmique, les microdomaines enrichis en cavéoline (MEC) [42]. Ce ciblage d'Akt dans les MEC empêche ainsi la kinase d'être recrutée à la membrane plasmique où elle est, normalement, activée en réponse à l'insuline $[43,44]$. Dans des cellules dépourvues de MEC, les céramides inhibent Akt par un autre mécanisme qui implique la phosphatase PP2A (protéine phosphatase 2A) qui déphosphoryle la kinase [44] (Figure 3). À plus long terme, les céramides ciblent et inhibent aussi les IRS par des mécanismes impliquant PKR/JNK (double-stranded RNA-dependent protein kinase/cJun kinase) et/ou Prepl ( $P b x$ regulating protein) et son cofacteur pl60, qui altèrent l'expression de GLUT4 et donc l'entrée de glucose dans la cellule $[45,46]$ (Figure 3). 


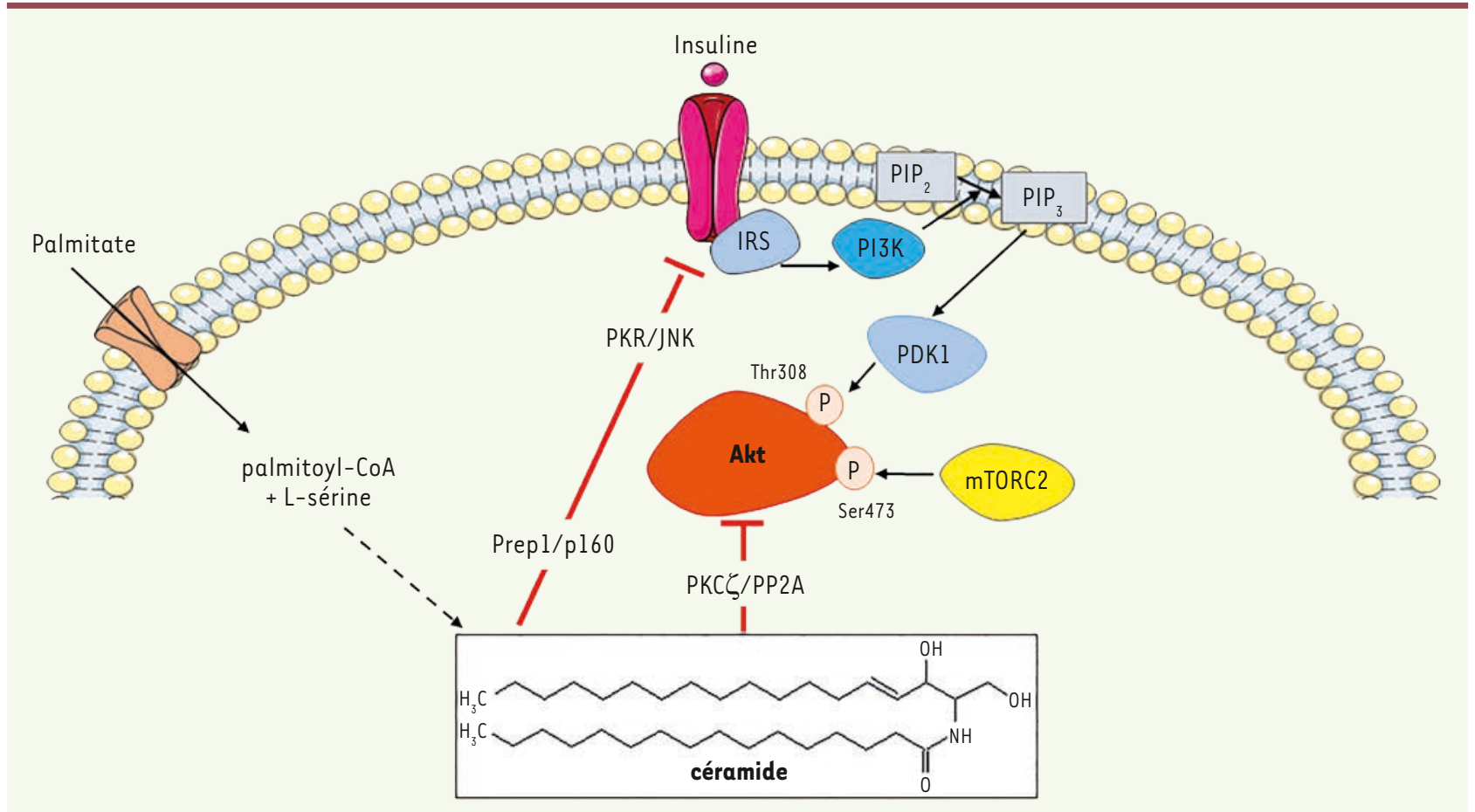

INSULINO-RÉSISTANCE

Figure 3. Action négative des céramides sur le signal de l'insuline dans les cellules. Les céramides inhibent la voie de signalisation de l'insuline en ciblant deux acteurs importants, Akt (protéine kinase B) et les IRS (insulin receptor substrates). Les céramides activent rapidement la PP2A (pro-

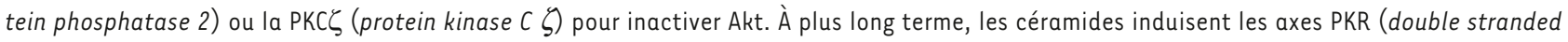
ARN-activated protein kinase)/JNK (c-Jun NH2-terminal kinase) et/ou Prepl (Pbx regulating protein 1)/pl60 pour cibler négativement les IRS.

\section{Autres dérivés sphingolipidiques impliqués dans l'insulino-}

\section{résistance}

Les dérivés sphingolipidiques synthétisés à partir des céramides peuvent aussi agir sur la signalisation insulinique dans les tissus. En effet, dans un modèle de souris, l'invalidation totale du gène codant Cerk, la céramide kinase responsable de la formation de céramide1-phosphate à partir de céramide, protège les animaux de l'obésité et de l'intolérance au glucose induites par un régime gras. Elle préserve également le tissu adipeux des souris d'une infiltration par les macrophages, empêchant ainsi l'inflammation de ce tissu [47].

Les gangliosides, une autre classe de sphingolipides dérivés de céramides, modulent également la signalisation insulinique, en particulier le GM3 (ganglioside monosialo 3). II a en effet été montré que la résistance à l'insuline induite par le TNF- $\alpha$ (tumor necrosis factor alpha) était associée à des concentrations élevées de GM3 dans les adipocytes [48], et que des souris invalidées pour le gène codant la GM3 synthase, et nourries avec un régime riche en graisse, présentaient une meilleure tolérance au glucose que des souris sauvages soumises au même régime [49]. L'augmentation des concentrations membranaires en GM3 favorise la dissociation du complexe que forme le récepteur de I'insuline avec la cavéoline 1 au niveau de la membrane, inhibant ainsi la propagation du signal insulinique $[50,51]$.

\section{Céramides, cibles thérapeutiques?}

L'implication des céramides dans le développement du DT2 est désormais bien établie. Cibler ces lipides pour prévenir leurs actions néfastes dans les cellules apparaît donc une approche thérapeutique intéressante pour améliorer la sensibilité à l'insuline [52]. Deux interventions peuvent être envisagées : 1) agir sur les protéines de signalisation,

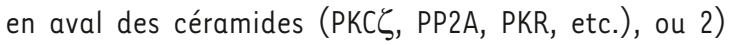
moduler les concentrations intracellulaires de céramides. Les protéines de signalisation que ciblent les céramides participent à de nombreuses autres voies que celles régulant la voie de signalisation insulinique. II semble donc difficile de moduler spécifiquement celles impliquées dans la voie insulinique sans affecter d'autres cibles secondaires.

La deuxième stratégie apparaît donc plus valide. Ainsi, chez des rongeurs soumis à un régime riche en graisse, l'inhibition pharmacologique, par la myriocine ${ }^{2}$ [53,

${ }^{2}$ La myriocine est un inhibiteur sélectif de la sérine palmitoyl-transférase, capable d'inhiber la formation de céramides. 
54] ou le fenrétinide [55], d'enzymes impliquées dans la voie de biosynthèse de novo des céramides (respectivement SPT [sérine palmitoyltransférase] et DESI [dihydrocéramide désaturase]), est suffisante pour améliorer la tolérance au glucose et la sensibilité à l'insuline. De même, une approche génétique a aussi confirmé le rôle des céramides dans la résistance à l'insuline in vivo. En effet, chez des souris diabétiques dont le gène codant DESI a été altéré (souris $\mathrm{DES}^{-/+}$), une diminution des concentrations tissulaires de céramides, accompagnée d'une amélioration de la sensibilité à l'insuline a été observée, par rapport à leurs compagnons de litière de type sauvage [56]. Ces études réalisées chez l'animal seront néanmoins difficilement envisageables chez l'homme en raison de l'importance des céramides comme colonne vertébrale des sphingolipides. II est en effet probable qu'inhiber complètement leur synthèse pourrait être dommageable pour les cellules. Le but étant de restaurer (ou de garder) des concentrations «physiologiques » intra-tissulaires de céramides, une possibilité pourrait être de cibler spécifiquement certaines espèces de céramides. Plusieurs études ont montré qu'en fonction du type cellulaire, des espèces de céramides différentes pouvaient être associées à la lipotoxicité (C16-céramides dans les hépatocytes, C18-céramides dans les cellules musculaires) (Figure 3). Inhiber la biosynthèse de ces espèces de céramides de façon tissu-spécifique, en utilisant des inhibiteurs ciblant les céramides synthases impliquées dans leur acylation pourrait donc être envisagé.

L'inhibition de la synthèse de certaines espèces de céramides pourrait présenter des effets néfastes sur la cellule. Les céramides agissent en effet comme des «lipides suppresseurs de tumeurs », en régulant de nombreuses réponses cellulaires fondamentales (apoptose, cycle cellulaire et autophagie) [57]. De même, chez la souris, l'inhibition de CerS2, qui induit une diminution des concentrations de C24-céramides, est associée à une fibrose hépatique sévère [58], à une augmentation de la perméabilité intestinale [59], et à une inflammation des voies respiratoires [60]. II sera donc important de vérifier que les inhibiteurs de céramide synthase potentiellement utilisés à des fins thérapeutiques n'entraîneront pas de tels effets indésirables.

La quantification des sphingolipides circulants pourrait être importante dans l'optique de suivre le développement d'un diabète, puisqu'il a été récemment montré que certains sphingolipides pouvaient être utilisés comme biomarqueurs afin d'identifier les individus à risque de développer un DT2. Les concentrations circulantes de dihydrocéramides (sphingolipides précurseurs des céramides) sont en effet significativement élevées chez les individus pré-diabétiques, jusqu'à 9 ans avant le début de la maladie [61]. Ces dihydrocéramides circulants sont de meilleurs biomarqueurs du DT2 que les céramides circulants [62]. Peu de données, à l'échelle cellulaire ou chez l'animal, existent sur le rôle mécanistique de ces dihydrocéramides sur la physiopathologie du DT2. Des études complémentaires seront donc nécessaires pour préciser l'intérêt de cette espèce de sphingolipides dérivée des céramides comme biomarqueur ou cible thérapeutique dans le DT2.

\section{Conclusion}

Au fil des années, de nombreuses études ont montré que les céramides jouaient un rôle crucial et délétère dans la régulation de la sensibilité à l'insuline tissulaire. Le décryptage des mécanismes par lesquels les céramides agissent négativement sur la signalisation de l'insuline a été un autre grand pas dans la compréhension de leur rôle. La priorité reste toutefois de déterminer avec certitude quelle(s) espèce(s) de céramide(s) est(sont) responsable(s) de l'insulino-résistance induite par la lipotoxicité dans les tissus cibles de l'hormone. Ces efforts seront cruciaux pour identifier finement des cibles thérapeutiques potentielles pour le développement de médicaments réduisant le risque de troubles associés aux lipides et de leurs maladies métaboliques et cardiovasculaires. $\diamond$

\section{SUMMARY}

Ceramides, crucial actors in the development of insulin resistance and type 2 diabetes

In healthy subjects, the balance between glucose production and its usage is precisely controlled. When circulating glucose reaches a critical threshold, pancreatic $\beta$-cells secrete insulin, which has two major actions: lowering circulating glucose concentrations by facilitating its uptake mainly in skeletal muscles and the liver, and inhibiting glucose production. Triglycerides are the main source of fatty acids to meet the energy needs of oxidative tissues and any excess is stored in adipocytes. Thus, adipose tissue acts as a trap for excess fatty acids released from plasma triglycerides. When the buffering action of adipose tissue to store fatty acids is impaired, they accumulate in other tissues where they are metabolized in several lipid species, including sphingolipid derivatives such as ceramides. Numerous studies have shown that ceramides are among the most active lipid second messengers to inhibit insulin signalling. This review describes the major role played by ceramides in the development of insulin resistance in peripheral tissues. $\diamond$

\section{LIENS D'INTÉRÊT}

Les auteurs déclarent n'avoir aucun lien d'intérêt concernant les données publiées dans cet article.

\section{RÉFÉRENCES}

1. Franc C. Le diabète : des chiffres alarmants. Med Sci (Paris) 2013 ; 29 : 711-4.

2. White JR. Economic considerations in treating patients with type 2 diabetes mellitus. Am J Health Syst Pharm 2002 ; 59 (suppl 9) : S14-7.

3. Williams EP, Mesidor M, Winters K, et al. Overweight and obesity: prevalence, consequences, and causes of a growing public health problem. Curr Obes Rep $2015 ; 4$ : 363-70. 


\section{RÉFÉRENCES}

4. Kwak SH, Park KS. Recent progress in genetic and epigenetic research on type 2 diabetes. Exp Mol Med $2016 ; 48:$ e220.

5. Petersen MC, Shulman GI. Mechanisms of insulin action and insulin resistance. Physiol Rev 2018 ; $98: 2133-23$.

6. Flamment $M$, Hajduch $\varepsilon$, Ferre $P$, et al. New insights into $\varepsilon R$ stress-induced insulin resistance. Trends Endocrinol Metab $2012 ; 23: 381-90$.

7. Randle PJ, Garland PB, Hales CN, et al. The glucose fatty-acid cycle. Its role in insulin sensitivity and the metabolic disturbances of diabetes mellitus. Lancet $1963 ; 1: 785-9$.

8. McGarry JD. Banting lecture 2001: dysregulation of fatty acid metabolism in the etiology of type 2 diabetes. Diabetes $2002 ; 51: 7-18$.

9. Hu M, Phan F, Bourron 0, et al. Steatosis and NASH in type 2 diabetes. Biochimie $2017 ; 143: 37-41$.

10. Boucher J, Kleinridders A, Kahn CR. Insulin receptor signaling in normal and insulin-resistant states. Cold Spring Harb Perspect Biol $2014 ; 6$.

11. Hajduch $\varepsilon$. Transport de glucose dans les tissus sensibles à l'insuline : implication de la protéine kinase B. Med Sci (Paris) $2001 ; 17$ : 1084-5.

12. Hage Hassan $R$, Bourron 0 , Hajduch $\varepsilon$. Defect of insulin signal in peripheral tissues: important role of ceramide. World J Diabetes $2014 ; 5: 244-57$.

13. Aon MA, Bhatt N, Cortassa SC. Mitochondrial and cellular mechanisms for managing lipid excess. Front Physiol $2014 ; 5: 282$.

14. Phillips DI, Caddy S, Ilic V, et al. Intramuscular triglyceride and muscle insulin sensitivity: evidence for a relationship in nondiabetic subjects. Metabolism $1996 ; 45: 947-50$

15. Thomas EL, Fitzpatrick JA, Malik SJ, et al. Whole body fat: content and distribution. Prog Nucl Magn Reson Spectrosc $2013 ; 73: 56-80$.

16. Hannun YA, Obeid LM. Principles of bioactive lipid signalling: lessons from sphingolipids. Nat Rev Mol Cell Biol $2008 ; 9$ : 139-50.

17. Hannun YA, Obeid LM. Sphingolipids and their metabolism in physiology and disease. Nat Rev Mol Cell Biol $2018 ; 19: 175-91$.

18. Mullen TD, Hannun YA, Obeid LM. Ceramide synthases at the centre of sphingolipid metabolism and biology. Biochem J $2012 ; 441: 789-802$

19. Turinsky J, 0'Sullivan DM, Bayly BP. 1,2-Diacylglycerol and ceramide levels in insulin-resistant tissues of the rat in vivo. J Biol Chem $1990 ; 265: 16880-5$.

20. Turinsky J, Bayly BP, O'Sullivan DM. 1,2-Diacylglycerol and ceramide levels in rat skeletal muscle and liver in vivo. Studies with insulin, exercise, muscle denervation, and vasopressin. J Biol Chem $1990 ; 265: 7933-8$.

21. Holland WL, Summers SA. Sphingolipids, insulin resistance, and metabolic disease: new insights from in vivo manipulation of sphingolipid metabolism. Endocr Rev $2008 ; 29$ : 381-402.

22. Bandet CL, Tan-Chen S, Bourron 0 , et al. Sphingolipid metabolism: new insight into ceramideinduced lipotoxicity in muscle cells. Int J Mol Sci 2019 ; 20 : ع479.

23. Adams JM, Pratipanawatr T, Berria R, et al. Ceramide content is increased in skeletal muscle from obese insulin-resistant humans. Diabetes $2004 ; 53: 25-31$.

24. Coen PM, Dube JJ, Amati F, et al. Insulin resistance is associated with higher intramyocellula triglycerides in type I but not type II myocytes concomitant with higher ceramide content. Diabetes $2010 ; 59: 80-8$

25. Moro C, Galgani JE, Luu L, et al. Influence of gender, obesity, and muscle lipase activity on intramyocellular lipids in sedentary individuals. J Clin Endocrinol Metab 2009 ; $94: 3440-7$

26. Dube JJ, Amati F, Toledo FG, et al. Effects of weight loss and exercise on insulin resistance, and intramyocellular triacylglycerol, diacylglycerol and ceramide. Diabetologia $2011 ; 54: 1147-56$

27. Blachnio-Zabielska AU, Koutsari C, Tchkonia T, et al. Sphingolipid content of human adipose tissue: relationship to adiponectin and insulin resistance. Obesity $2012 ; 20: 2341-7$.

28. Yu C, Chen Y, Cline GW, et al. Mechanism by which fatty acids inhibit insulin activation of insulin receptor substrate-1 (IRS-1)-associated phosphatidylinositol 3-kinase activity in muscle. J Biol Chem $2002 ; 277: 50230-6$.

29. Itani SI, Ruderman NB, Schmieder F, et al. Lipid-induced insulin resistance in human muscle is associated with changes in diacylglycerol, protein kinase $C$, and IKB- $\alpha$. Diabetes $2002 ; 51: 2005-11$.

30. Vistisen B, Hellgren LI, Vadset $\mathrm{T}$, et al. Effect of gender on lipid-induced insulin resistance in obese subjects. Eur J Endocrinol $2008 ; 158: 61-8$.

31. Kotronen A, Seppanen-Laakso T, Westerbacka J, et al. Hepatic stearoyl-CoA desaturase (SCD)-1 activity and diacylglycerol but not ceramide concentrations are increased in the nonalcoholic human fatty liver. Diabetes $2009 ; 58: 203-8$.

32. Magkos F, Su X, Bradley D, et al. Intrahepatic diacylglycerol content is associated with hepatic insulin resistance in obese subjects. Gastroenterology 2012 ; 142 : 1444-6.

33. Raichur S, Wang ST, Chan PW, et al. CerS2 haploinsufficiency inhibits beta-oxidation and confers susceptibility to diet-induced steatohepatitis and insulin resistance. Cell Metab $2014 ; 20: 687-95$

34. Chaurasia B, Tippetts TS, Mayoral Monibas R, et al. Targeting a ceramide double bond improves insulin resistance and hepatic steatosis. Science $2019 ; 365: 386-92$

35. Turpin SM, Nicholls HT, Willmes DM, et al. Obesity-induced CerS6-dependent C16:0 ceramide production promotes weight gain and glucose intolerance. Cell Metab $2014 ; 20: 678$-86.

36. Jiang M, Li C, Liu Q, et al. Inhibiting ceramide synthesis attenuates hepatic steatosis and fibrosis in rats with non-alcoholic fatty liver disease. Front Endocrinol 2019; $10: 665$.

37. Matsuzaka T, Kuba M, Koyasu S, et al. Hepatocyte Elovl6 determines ceramide acyl-chain length and hepatic insulin sensitivity in mice. Hepatology 2019 ; Sep 17. doi: 10.1002/hep.30953.
38. Kurek K, Piotrowska DM, Wiesiolek-Kurek P, et al. Inhibition of ceramide de novo synthesis reduces liver lipid accumulation in rats with nonalcoholic fatty liver disease. Liver Int 2014 ; 34 : 1074-83.

39. Raichur S, Brunner B, Bielohuby $\mathrm{M}$, et al. The role of $\mathrm{Cl6:0}$ ceramide in the development of obesity and type 2 diabetes: CerS6 inhibition as a novel therapeutic approach. Mol Metab $2019 ; 21: 36-50$.

40. Bergman BC, Brozinick JT, Strauss A, et al. Muscle sphingolipids during rest and exercise: a C18:0 signature for insulin resistance in humans. Diabetologia 2016 ; 59 : 785-98.

41. Turpin-Nolan SM, Hammerschmidt P, Chen W, et al. CerS1-derived C18:0 ceramide in skeletal muscle promotes obesity-induced insulin resistance. Cell Rep $2019 ; 26: 1-10$.

42. Hajduch $\varepsilon$, Turban S, Le L, et al. Targeting of PKCzeta and PKB to caveolinenriched microdomains represents a crucial step underpinning the disruption in PKB-directed signalling by ceramide. Biochem / $2008 ; 410: 369-79$.

43. Powell D, Hajduch $\varepsilon$, Kular G, et al. Ceramide disables 3 -phosphoinositide binding to the pleckstrin homology domain of protein kinase B (PKB)/Akt by a PKC zeta-dependent mechanism. Mol Cell Biol $2003 ; 23$ : 7794-808.

44. Blouin CM, Prado C, Takane KK, et al. Plasma membrane subdomain compartmentalization contributes to distinct mechanisms of ceramide action on insulin signaling. Diabetes $2010 ; 59: 600-10$.

45. Hage Hassan R, Pacheco de Sousa AC, Mahfouz R, et al. Sustained action of ceramide on the insulin signaling pathway in muscle cells: implication of the double-stranded RNA-activated protein kinase. J Biol Chem 2016 ; 291 : 3019-29.

46. Cimmino I, Lorenzo V, Fiory F, et al. A peptide antagonist of Prepl-pl60 interaction improves ceramide-induced insulin resistance in skeletal muscle cells. Oncotarget $2017 ; 8: 71845-58$

47. Mitsutake S, Date T, Yokota $\mathrm{H}$, et al. Ceramide kinase deficiency improves diet-induced obesity and insulin resistance. FEBS Lett $2012 ; 586: 1300-5$.

48. Tagami S, Inokuchi JJ, Kabayama K, et al. Ganglioside GM3 participates in the pathological conditions of insulin resistance. J Biol Chem 2002 ; 277 : 3085-92.

49. Yamashita T, Hashiramoto A, Haluzik M, et al. Enhanced insulin sensitivity in mice lacking ganglioside GM3. Proc Natl Acad Sci USA 2003 ; 100 : 3445-9.

50. Sekimoto J, Kabayama K, Gohara K, et al. Dissociation of the insulin receptor from caveolae during TNFalpha-induced insulin resistance and its recovery by D-PDMP. FEBS Lett $2012 ; 586: 191-5$.

51. Kabayama K, Sato T, Saito K, et al. Dissociation of the insulin receptor and caveolin- 1 complex by ganglioside GM3 in the state of insulin resistance. Proc Natl Acad Sci USA 2007 ; 104 : 13678-83.

52. Hajduch $\varepsilon$, Bourron 0 . Type 2 diabetes: ceramides as a therapeutic target? Clin Lipidol $2013 ; 8: 607-9$

53. Ussher JR, Koves TR, Cadete VJ, et al. Inhibition of de novo ceramide synthesis reverses diet-induced insulin resistance and enhances whole-body oxygen consumption. Diabetes $2010 ; 59: 2453-64$

54. Blachnio-Zabielska AU, Chacinska M, Vendelbo MH, et al. The crucial role of $\mathrm{Cl}$-Cer in fat-induced skeletal muscle insulin resistance. Cell Physiol Biochem 2016; $40: 1207-20$.

55. Bikman BT, Guan Y, Shui G, et al. Fenretinide prevents lipid-induced insulin resistance by blocking ceramide biosynthesis. J Biol Chem 2012 ; 287 : 17426-37.

56. Holland WL, Brozinick JT, Wang LP, et al. Inhibition of ceramide synthesis ameliorates glucocorticoid-, saturated-fat-, and obesity-induced insulin resistance. Cell Metab $2007 ; 5: 167-79$

57. Morad SA, Cabot MC. Ceramide-orchestrated signalling in cancer cells. Nat Rev Cancer $2013 ; 13: 51-65$.

58. Grammatikos G, Ferreiros N, Bon D, et al. Variations in serum sphingolipid levels associate with liver fibrosis progression and poor treatment outcome in hepatitis C virus but not hepatitis B virus infection. Hepatology $2015 ; 61: 812-22$.

59. Kim YR, Volpert $G$, Shin KO, et al. Ablation of ceramide synthase 2 exacerbates dextran sodium sulphate-induced colitis in mice due to increased intestinal permeability. J Cell Mol Med 2017 ; 21 : 3565-78.

60. Petrache I, Kamocki K, Poirier C, et al. Ceramide synthases expression and role of ceramide synthase- 2 in the lung: insight from human lung cells and mouse models. PLoS One 2013; 8 : e62968.

61. Wigger L, Cruciani-Guglielmacci C, Nicolas A, et al. Plasma dihydroceramides are diabetes susceptibility biomarker candidates in mice and humans. Cell Rep 2017 ; 18 : 2269-79.

62. Szpigel A, Hainault I, Carlier A, et al. Lipid environment induces ER stress, TXNIP expression and inflammation in immune cells of individuals with type 2 diabetes. Diabetologia $2018 ; 61: 399-412$

\section{TIRÉS À PART}

६. Hajduch 